\title{
Contrast Harmonic Imaging of Canine Hepatic Tumors
}

\author{
Kenji KUTARA $^{1)}$, Kazushi ASANO ${ }^{1) *}$, Ayako KITO $^{1)}$, Kenji TESHIMA ${ }^{1)}$, Yuka KATO ${ }^{1)}$, Yukie SASAKI ${ }^{1)}$, \\ Kazuya EDAMURA $^{1)}$, Hisashi SHIBUYA ${ }^{2)}$, Tsuneo SATO $^{2)}$, Atsuhiko HASEGAWA ${ }^{3)}$ and Shigeo TANAKA ${ }^{1)}$ \\ ${ }^{1)}$ Laboratories of Veterinary Surgery, ${ }^{2)}$ Veterinary Pathology and ${ }^{3)}$ Veterinary Pathobiology, Department of Veterinary Medicine, College \\ of Bioresource Sciences, Nihon University, Kameino 1866, Fujisawa 252-8510, Japan
}

(Received 30 September 2005/Accepted 11 January 2006)

ABSTRACT. Six adult healthy Beagles were used to investigate the hepatic perfusion dynamics of Levovist, a contrast agent used in contrast harmonic imaging (CHI). In addition, 8 dogs with hepatocellular carcinoma (HCC) and 2 dogs with metastatic hepatic hemangiosarcoma (HSA) were used to characterize both the CHI findings with Levovist. In the Beagles, the start of intravenously injected Levovist into the aorta between the cranial mesenteric and renal arteries and the portal vein at the hepatic hilum were $5.47 \pm 1.52 \mathrm{sec}$ and $16.03 \pm$ $3.39 \mathrm{sec}$, respectively. As a characteristic CHI finding in the $8 \mathrm{dogs}$ with $\mathrm{HCC}$, the early arterial phase showed a fine network of blood flow enhanced at the surrounding region and within the tumor in all the $8 \mathrm{dogs}(100 \%)$, and the post vascular phase demonstrated a defect in the whole tumor and an enhancement of the surrounding hepatic tissues in 7 dogs $(87.5 \%)$. In the 2 dogs with HSA, characteristic finding in which the early arterial and late vascular phases showed a rim contrast enhancement pattern, and the post vascular phase revealed that the whole tumor lacked contrast enhancement and the surrounding hepatic tissues was clearly enhanced. In dogs, the start of the early arterial and late vascular phases, and the characterizations of the CHI findings in HCC and HSA were suggested to be similar to those in humans. Therefore, $\mathrm{CHI}$ is thought to be useful for the diagnosis of HCC and metastatic hepatic HSA in dogs as well as in humans.

KEY WORDS: canine, contrast harmonic imaging, hemangiosarcoma, hepatocellular carcinoma, Levovist.

J. Vet. Med. Sci. 68(5): 433-438, 2006

Contrast harmonic imaging (CHI) is the state of the art in diagnostic ultrasound for the evaluation of tissue perfusion in various ischemic diseases and vascularity in tumors. CHI is achieved by the intravenous administration of microbubbles, which pass through the pulmonary capillary beds. Each characteristic vascularity pattern in human hepatic benign and malignant lesions is detectable because intravenous microbubbles produce a more potent harmonic signal by the transmitted ultrasound beam than that produced by the surrounding tissue. It has been demonstrated that $\mathrm{CHI}$ provides a large variety of important information on the diagnosis, malignancy, therapeutic response, and prognosis in human hepatic tumors [2-4, 6, 7, 9, 11, 13-15].

Levovist (Schering Co., Berlin, Germany) is an ultrasound contrast medium and is composed of galactose $(99.9 \%)$ and palmitic acid (0.1\%). A suspension of Levovist with sterilized water contains air microbubbles with a mean diameter of $3 \mu \mathrm{m}$. Echogenic enhancement of blood flow begins within seconds after an intravenous bolus injection of the suspension and lasts for a time period of $5 \mathrm{~min}[5,15]$. The hepatic CHI using Levovist is then classified into 3 different phases: an early arterial phase corresponding to arterial blood flow, a late vascular phase comprising late arterial and portal blood flows, and a post vascular phase reflecting the accumulation of microbubbles in the liver parenchyma (sinusoidal and/or Kupffer cells) [7, 15]. The characterization of the different phases of CHI in the human liver has

\footnotetext{
* Correspondence to: Asano, K., Laboratory of Veterinary Surgery, Department of Veterinary Medicine, College of Bioresource Sciences, Nihon University, Kameino 1866, Fujisawa, Kanagawa 252-8510, Japan.
}

been demonstrated to be of clinical importance for the differential diagnosis of hepatic benign and malignant focal lesions such as hepatocellular carcinoma (HCC), hemagioma, metastatic sarcoma/carcinoma, and focal nodular hyperplasia [2, 7, 9].

However, to the best of our knowledge, a limited number of veterinary studies on canine $\mathrm{CHI}$ have been published $[10,12,17]$. We believe that $\mathrm{CHI}$ is of value in small animal medicine, similar to humans, considering that it is a convenient and noninvasive method that does not involve sedation/anesthesia for the differential diagnosis of hepatic malignant tumors. In particular, CHI may possess the potential for playing an important role in the determination of surgical indication in the case of hepatic focal diseases. The aim of this study was to investigate the hepatic perfusion dynamics of Levovist in canine normal liver and characterize CHI findings in naturally occurring $\mathrm{HCC}$ and metastatic hepatic hemangiosarcoma (HSA) in dogs.

\section{MATERIALS AND METHODS}

Animals: Six adult Beagles (5 males and 1 female, weight: $10.22-12.52 \mathrm{~kg}$ ) were used for the determination of early arterial and late vascular phases in canine normal hepatic CHI. The Beagles were confirmed to be healthy based on the results of the general physical examination, complete blood cell counts, and general serum biochemical profile. Abdominal ultrasound did not reveal hepatic focal lesions in any of the Beagles. All dogs were cared for according to the principles outlined in the guide for the Care and Use of Laboratory Animals approved by the College of 
Bioresource Sciences, Nihon University.

Eight privately owned dogs were used for the characterization of the CHI findings in clinical cases of canine HCC and metastatic hepatic tumor. The dogs were referred to the Animal Medical Center of Nihon University with suspicion of hepatic or splenic tumor. A temporary diagnosis of HCC was derived from abdominal radiography, ultrasound and/or computed tomography (CT), and tru-cut biopsy from 8 dogs (2 Shibas, 2 Yorkshire Terriers, 1 Shuh Tzu, 1 Standard Poodle, 1 Golden Retriever and 1 Mongrel). A temporary diagnosis of splenic HSA was obtained from abdominal radiography and ultrasound in the other 2 dogs (Golden Retrievers). All dogs were used for the CHI prior to the exploratory laparotomy.

Preparation of Levovist: A vial of Levovist contains $2.4975 \mathrm{~g}$ of galactose and $0.0025 \mathrm{~g}$ of palmitic acid. The agent $(2.5 \mathrm{~g})$ was shaken for approximately $10 \mathrm{sec}$ with $7 \mathrm{ml}$ of sterile distilled water to yield an opalescent suspension of air microbubbles $(300 \mathrm{mg} / \mathrm{m} \mathrm{l}$ of the contrast agent; approximately $8.5 \mathrm{~m} l$ in total). Two minutes after the suspension was equilibrated, Levovist could be used for the bolus injection.

Determination of early arterial and late vascular phases in canine normal liver (experiment 1): A 22 G over-the-needle catheter was placed into the cephalic vein of the Beagles for the administration of anesthetics and contrast agents. Each dog was premedicated with midazolam hydrochloride $(0.2 \mathrm{mg} / \mathrm{kg}$, intravenous $)$ and butorphanol tartrate $(0.2 \mathrm{mg} /$ $\mathrm{kg}$, intravenous) and was intubated after anesthesia was induced by thiopental sodium (15 $\mathrm{mg} / \mathrm{kg}$, intravenous). General anesthesia was maintained with inhalation of isoflurane $(1.5 \%)$ and oxygen $(2 \mathrm{l} / \mathrm{min})$ under mechanical ventilation (respiratory rate, 10 breaths/min; respiratory pressure, 10 to $15 \mathrm{cmH}_{2} \mathrm{O}$; end-expiratory carbon dioxide concentration, $40 \mathrm{mmHg}$ ) with intravenous bolus administration of pancuronium bromide $(0.04$ to $0.05 \mathrm{mg} / \mathrm{kg})$, if necessary.

Each dog was positioned in dorsal recumbency. A diagnostic ultrasound system, Aplio (Toshiba Medical Systems, Tokyo, Japan), along with a 3-6 MHz broadband convex transducer, was used; all images were derived from optimized settings of CHI software developed by the manufacturer. Transverse plane at the level of the right renal cranial pole with a characteristic appearance of the aorta (Ao) between the cranial mesenteric artery and the renal artery was used for the estimation of the early arterial phase. The region of interest (ROI) with a diameter of $3 \mathrm{~mm}$ is allocated within Ao in transverse imaging. Immediately after the administration of $0.1 \mathrm{~m} / / \mathrm{kg}$ of Levovist via a 3-way stopcock with a $50-\mathrm{cm}$ long extension tube connected into the indwelling cephalic vein, the time intensity curve (TIC) of the echogenic level in the ROI is recorded for a minute at the rate of 5 frames per second (Fig. 1a).

Next, the position of the dogs was changed to left lateral recumbency. Transverse plane at the level of the hepatic hilum in which the main portal vein (PV) was being fed into the liver was used for the estimation of the late vascular phase. ROI with a diameter of $3 \mathrm{~mm}$ is allocated within PV in transverse imaging. Immediately after the administration of $0.1 \mathrm{~m} l / \mathrm{kg}$ of Levovist, the TIC of the echogenic level in the ROI is recorded, as described previously (Fig. 1b).

Characterization of $\mathrm{CHI}$ findings in canine $\mathrm{HCC}$ and metastatic hepatic tumor (experiment 2): A 22 to $24 \mathrm{G}$ overthe-needle catheter was placed into the cephalic vein in the dogs. Each dog was positioned in dorsal recumbency. A diagnostic ultrasound system, Nemio (Toshiba Medical Systems, Tokyo, Japan), with a 3-6 MHz broadband convex transducer was used, and all images were derived from the optimized settings of CHI software developed by the manufacturer. First, transverse and/or longitudinal planes of the hepatic focal lesion or the parenchyma in each dog were recorded for $1 \mathrm{~min}$ after the administration of Levovist in order to determine the characterization of $\mathrm{CHI}$ in the early arterial and late vascular phases. The start of the early arterial and late vascular phases were determined on the basis of experiment 1 . CHI had been stopped for $4 \mathrm{~min}$ after the first recording. From $5 \mathrm{~min}$ after the injection of Levovist, the $\mathrm{CHI}$ recording in the same plane was continued for $1 \mathrm{~min}$ in order to determine the characterization of $\mathrm{CHI}$ in the post vascular phase. The start of the post vascular phase used in this series, 5 min after the injection of Levovist, was extrapolated from that fixed in human hepatic CHI methodology [10]. All CHI data were recorded at a rate of frames per second by means of the flush echo imaging technique.

All dogs were operated for removal of the tumor after the
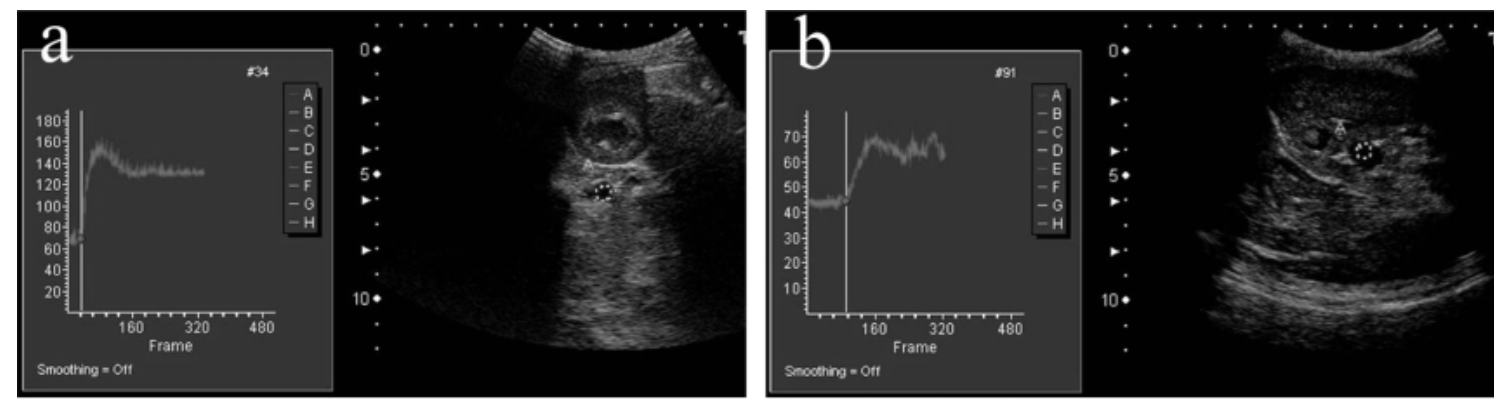

Fig. 1. Time intensity curves of the echogenic level derived from the region of interest in an aorta (a) between the cranial mesenteric artery and the renal artery for estimation of early arterial phase, and the region of interest in a portal vein (b) at the level of the hepatic hilum for estimation of late vascular phase. 
examination, and were definitively diagnosed as HCC or HSA based on the histological examination of the resected tumors after the operation.

Data analysis: In experiment 1 , the following were measured from the TIC data derived from the ROI of Ao and PV: time from the end of the bolus administration of Levovist $\left(\mathrm{T}_{0}\right)$ to the start of the upslope phase of TIC $\left(\mathrm{T}_{\text {up }}\right)$ and time from $\mathrm{T}_{0}$ to the peak upslope phase of TIC $\left(\mathrm{T}_{\text {peak }}\right)$. All data were expressed as mean \pm standard deviation (SD). The start of the early arterial and the late vascular phases were estimated from $\mathrm{T}_{\text {up }}$ in Ao and PV, respectively. Simple linear regression analysis was used to investigate the influence of body weight and heart rate on $\mathrm{T}_{\text {up }}$ and $\mathrm{T}_{\text {peak }}$. Statistical significance was defined as a $p$ value that was less than 0.05 .

\section{RESULTS}

Experiment 1: CHI data in the ROI of Ao and PV were obtained from all the beagles (Table 1). $\mathrm{T}_{\text {up }}$ and $\mathrm{T}_{\text {peak }}$ in the ROI of Ao were $5.47 \pm 1.52 \mathrm{sec}$ and $13.50 \pm 3.37 \mathrm{sec}$, respectively (Fig. 1a). The $\mathrm{T}_{\text {up }}$ and $\mathrm{T}_{\text {peak }}$ in the ROI of Ao were not correlated with the body weight and heart rate.

$\mathrm{T}_{\text {up }}$ and $\mathrm{T}_{\text {peak }}$ in the ROI of PV were $16.03 \pm 3.39 \mathrm{sec}$ and $27.50 \pm 4.09 \mathrm{sec}$, respectively (Fig. 1b). The $\mathrm{T}_{\text {up }}$ and $\mathrm{T}_{\text {peak }}$ in the ROI of PV had no correlation with the body weight and heart rate.
Experiment 2: In the 8 dogs with $\mathrm{HCC}$, the contrast enhancement patterns varied on an individual basis (Table 2 ). In the early arterial phase, 6 dogs $(75 \%)$ clearly showed a fine network of blood flow that was enhanced in the surrounding region and within the tumor (Figs. 2a and 3a). These findings were a basket-like enhancement pattern similar to that typically observed in human HCC. Such contrast enhancement pattern was partly observed in the 2 remaining dogs $(25 \%)$. Thus, the 8 dogs $(100 \%)$ indicated characteristic $\mathrm{CHI}$ findings in the early arterial phase. In the late vascular phase, 4 dogs $(50 \%)$ showed whole enhancement in the tumor (Figs. 2b and 3b), and another dog (12.5\%) revealed partial enhancement in the tumor. In contrast, there was no enhancement in the remaining 3 dogs (37.5\%). In the post vascular phase, 7 dogs $(87.5 \%)$ demonstrated a defect in the whole tumor and an enhancement of the surrounding hepatic tissues (Figs. 2c and 3c). However, there was no differentiation between the tumor and the surrounding hepatic tissues in the remaining $1 \mathrm{dog}(12.5 \%)$. In addition, each characteristic $\mathrm{CHI}$ imaging of all the 3 phases was consistently detected in $4(50.0 \%)$ of the 8 dogs (Table 2$)$.

In the 2 dogs with metastatic hepatic HSA, CHI findings in the early arterial and late vascular phases showed rim contrast enhancement pattern. In the post vascular phase, the whole tumor lacked contrast enhancement, and the surrounding hepatic tissues were clearly enhanced (Fig. 4).

Table 1. Data derived from time intensity curve of contrast harmonic imaging in experiment 1

\begin{tabular}{|c|c|c|c|c|c|c|}
\hline \multirow{2}{*}{$\begin{array}{l}\text { BW } \\
(\mathrm{kg})\end{array}$} & \multicolumn{3}{|c|}{ Aorta } & \multicolumn{3}{|c|}{ Portal Vein } \\
\hline & $\mathrm{T}_{\text {up }}(\mathrm{sec})$ & $\mathrm{T}_{\text {peak }}(\mathrm{sec})$ & HR (bpm) & $\mathrm{T}_{\text {up }}(\mathrm{sec})$ & $\mathrm{T}_{\text {peak }}(\mathrm{sec})$ & HR (bpm) \\
\hline $11.8 \pm 0.9$ & $5.47 \pm 1.52$ & $13.50 \pm 3.37$ & $89.6 \pm 13.9$ & $16.03 \pm 3.39$ & $27.50 \pm 4.09$ & $97.9 \pm 28.8$ \\
\hline
\end{tabular}

Table 2. Details of contrast harmonic imaging in 8 dogs with hepatocellular carcinoma

\begin{tabular}{|c|c|c|c|c|c|c|c|c|}
\hline No. & Breed & Age & Gender & Liver lobe $^{1)}$ & $\begin{array}{l}\text { Tumor size } \\
\text { (mm) }\end{array}$ & $\begin{array}{c}\text { Early arterial } \\
\text { phase }^{2)}\end{array}$ & $\begin{array}{c}\text { Late vascular } \\
\text { phase }^{3)}\end{array}$ & $\begin{array}{c}\text { Post vascular } \\
\text { phase }^{4)}\end{array}$ \\
\hline 1 & Yorkshire Terrier & 14 & Male & QL & $17.5 \times 27.7$ & + & + & + \\
\hline 2 & Shiba & 12 & Female & LLL & $68.4 \times 51.4$ & + & - & + \\
\hline 3 & Shiba & 14 & Male & RLL & $25.0 \times 26.7$ & + & + & + \\
\hline 4 & Mongrel & 12 & Male & RLL & $75.1 \times 48.3$ & + & + & + \\
\hline 5 & Shuh Tzu & 9 & Female & $\mathrm{CL}$ & $50.9 \times 65.5$ & + & - & + \\
\hline 6 & Golden Retriever & 7 & Female & LLL & $49.6 \times 36.5$ & + & + & - \\
\hline 7 & Yorkshire Terrier & 6 & Male & QL & $30.0 \times 33.6$ & \pm & - & + \\
\hline 8 & Standard Poodle & 9 & Female & LLL & $114.5 \times 107.7$ & \pm & \pm & + \\
\hline
\end{tabular}

Abbreviations: QL, quadrate lobe; LLL, left lateral lobe; RLL, right lateral lobe; CL, caudate lobe.

1) Liver lobe from which tumor originated.

2) Characteristic finding of contrast harmonic imaging in early arterial phase- a fine network enhancement of blood flow around and within the tumor (basket-like enhanced pattern): +, clearly observed; \pm partly observed; -, not observed.

3) Characteristic finding of contrast harmonic imaging in late vascular phase- whole enhancement in the tumor: + , clearly observed; \pm partly observed; -, not observed.

4) Characteristic finding of contrast harmonic imaging in post vascular phase- a defect in the whole tumor and an enhancement of the sorrounding hepatic tissue: +, clearly observed; \pm , partly observed; -, not observed. 

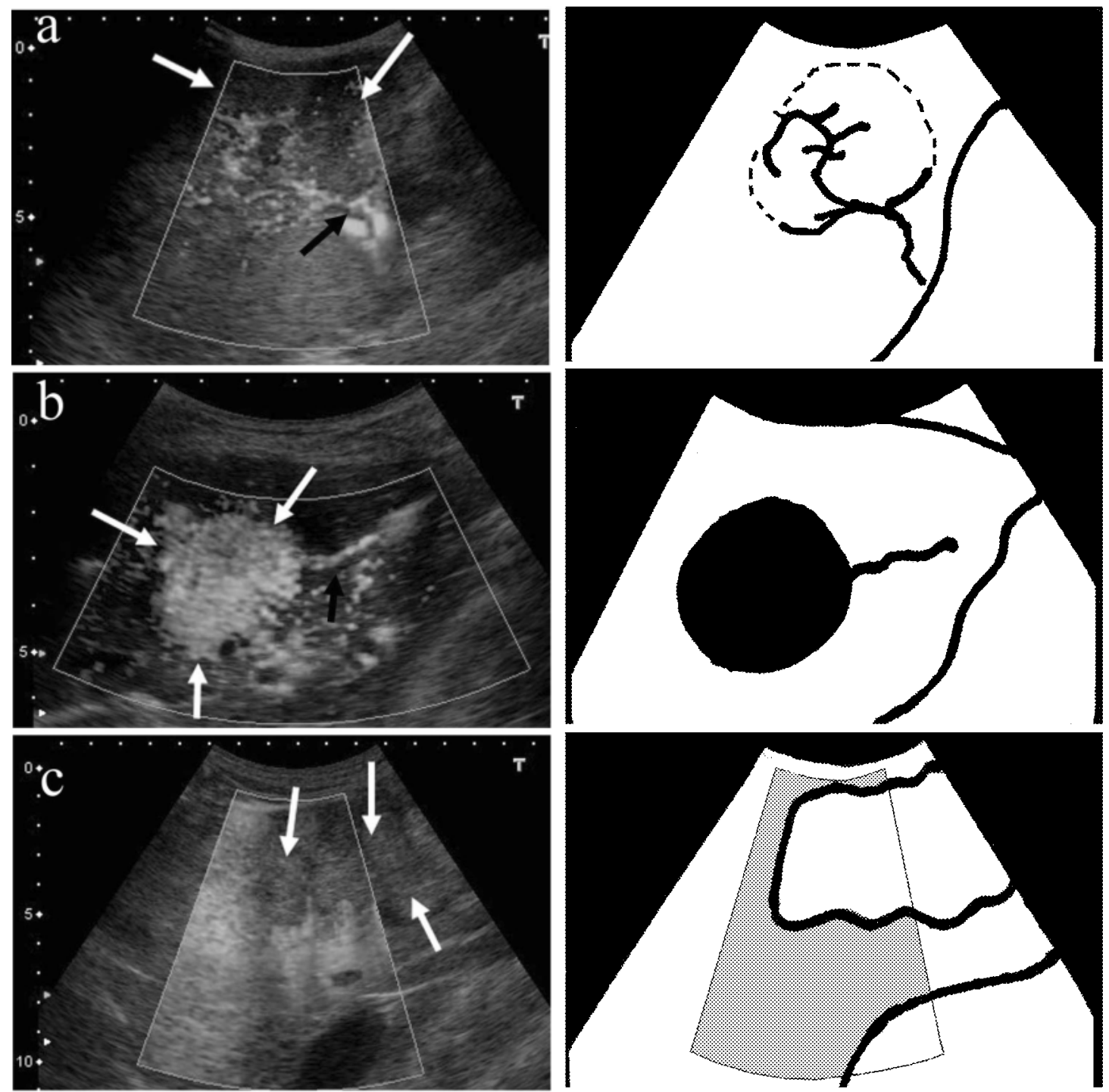

Fig. 2. Typical contrast enhancement pattern of canine hepatocellular carcinoma (HCC). Left-sided figures are contrast harmonic images in three different phases, and each right-sided figure shows a diagram of the left-side image. Typical contrast enhancement pattern (a) in the early arterial phase. White arrows indicate HCC. Enhanced arterial blood flow feeding from the surrounding region into the tumor was observed (black arrow). A fine network of blood flow within the tumor was developing. Typical contrast enhancement pattern (b) in the late vascular phase. White arrows indicate HCC. The finding showed whole tumor enhancement pattern. Enhanced blood flow feeding into the tumor was observed (black arrow). Typical contrast enhancement pattern (c) in the post vascular phase. White arrows indicate HCC. A defect in the whole tumor and an enhancement of the surrounding hepatic tissues were observed.

\section{DISCUSSION}

In experiment 1 , ROI was restricted to the abdominal Ao between the cranial mesenteric artery and the renal artery for the evaluation of the beginning of the early arterial phase in order to determine the different CHI phases in dogs. Earlier, it was proposed that ROI was located within the hepatic artery in the liver parenchyma; however, the appropriate size of the ROI could not be configured in the intrahepatic arterial branches due to their thinness. Hence, the ROI was finally located into the Ao between the cranial mesenteric artery and the renal artery. On the other hand, the ROI could be configured into the main PV at the level of the hepatic hilum near the hepatic parenchyma for the evaluation of the beginning of the late vascular phase. In either location, the true time at which the contrast agent passes into the liver after the intravenous injection might slightly be prolonged when compared with the time of start derived from experiment 1 . Although it is defined in human cases that the early arterial phase begins approximately $10 \mathrm{sec}$ and the late vas- 

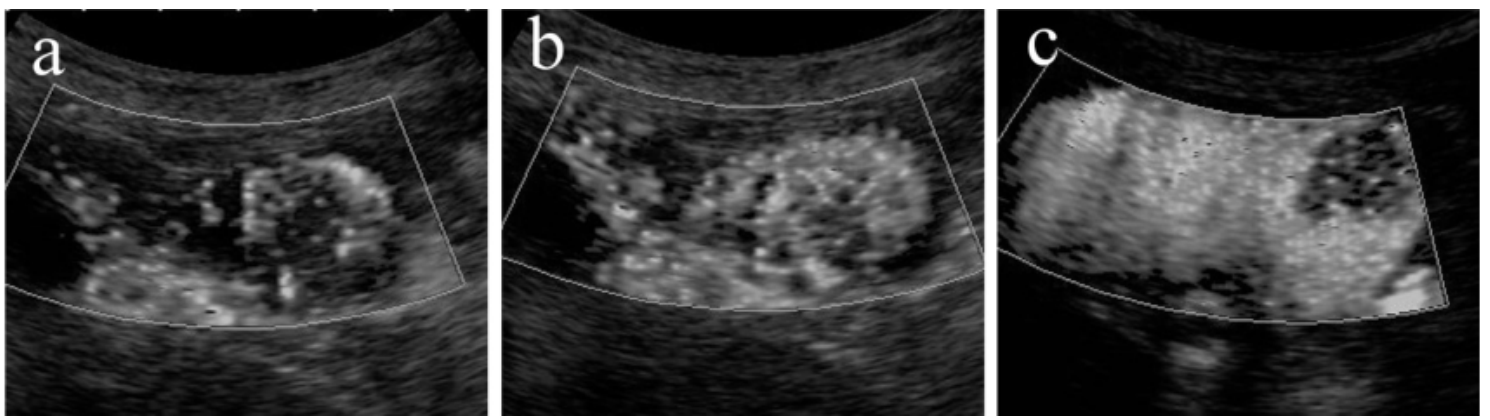

Fig. 3. Contrast harmonic imaging of early arterial (a), late vascular (b) and post vascular (c) phases in dog No. 1 with hepatocellular carcinoma. In the early arterial phase, blood flow were enhanced around the tumor. Afterwards, the whole tumor was enhanced in the late vascular phase. In the post vascular phase, a defect in the whole tumor and an enhancement of the surrounding hepatic tissue were observed.
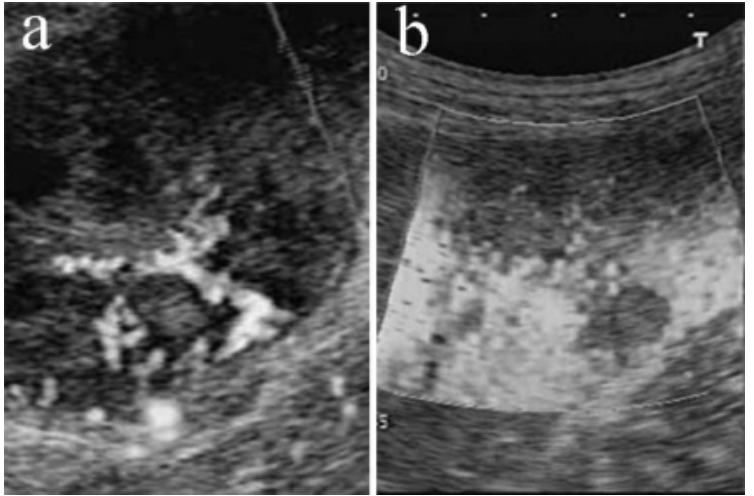

Fig. 4. Typical contrast enhancement pattern of metastatic hepatic hemangiosarcoma. The observations in the early arterial and late vascular phases (a) showed rim contrast enhancement pattern. In the post vascular phase (b), it was shown as a whole tumor defect pattern.

cular phase starts approximately $30 \mathrm{sec}$ after the injection of Levovist [13], the start of their phases in dogs might be defined similar to that in the humans. Although the start of the post vascular phase was not validated in our study, it was believed to set 5 min after the injection of Levovist similar to that in the humans. In fact, the characteristic CHI findings similar to those in the human cases in the post vascular phase of canine HCC and HSA were observed in almost all the cases in experiment 2. However, further investigations on the validation of the start of the post vascular phase are believed to be necessary. In addition, although the start of the early arterial and late vascular phases were not correlated with the body weight and the heart rate in experiment 1 , further studies of canine cases on a larger scale may be required to clarify whether the breed, body weight, and heart rate have an impact on the start of the three different phases in dogs.

In experiment 2, Typical CHI findings of the human $\mathrm{HCC}$ have been characterized into the following contrast patterns: early arterial phase indicates a fine network of blood flow with an enhancement in the border of the tumor; the late vas- cular phase shows whole enhancement in the tumor; and the post vascular phase indicates a defect in the whole tumor and opposite enhancement of the surrounding hepatic tissues $[2-4,6,11,14]$. The sensitivity and specificity of CHI have been demonstrated to be $68 \%-93 \%$ and $74 \%-100 \%$, respectively $[2-4,6,11,14]$. The results of canine HCC in experiment 2 revealed characteristic $\mathrm{CHI}$ findings similar to those of humans in all the three phases; these findings were consistently derived from 4 dogs $(50 \%)$. However, in the case of confinement to the early arterial phase, a fine enhanced network of arterial blood flow in the surrounding region and within the tumor, similar to those in humans, was ascertained in all the dogs, including the findings that were clearly observed in 6 dogs and partially in 2 dogs. Similarly, in the case that the post vascular phase was focused, the 7 dogs $(87.5 \%)$ showed a defect in the whole tumor and an enhancement of the surrounding hepatic tissues. The characteristic CHI findings of canine HCC may thus be easily derived from two phases (early arterial and post vascular phases). On the other hand, the typical $\mathrm{CHI}$ findings of HSA have been characterized as a contrast enhancement pattern of the rim of the tumor in the early arterial and late vascular phases, and a defect in the contrast pattern of the complete tumor in the post vascular phase $[2,11]$. The sensitivity and specificity of $\mathrm{CHI}$ have been shown to be $83.3 \%-98 \%$ and $77 \%-94.3 \%$, respectively $[2,11]$. Even though there were the only 2 dogs with HSA in experiment 2 , the findings were similar to those of the human cases. Therefore, $\mathrm{CHI}$ is believed to be clinically useful for the diagnosis of HCC and metastatic hepatic HSA in dogs as well as in humans.

To our knowledge, there is only one veterinary study that is reported in literature that has demonstrated $\mathrm{CHI}$ findings in 1 dog with $\mathrm{HCC}$ and in 3 dogs with metastatic hepatic HSA [10]. In the previous report, Definity was used as the contrast medium. Levovist consists of a galactose-based air-filled microbubble, whereas Definity is composed of a perfluoropropane gas-filled lipid-stabilized microbubble. Due to the different behaviors, Levovist is accumulated in Kupffer cells during the post vascular phase in contrast to Definity. In addition, Levovist was shown to have hepatic 
parenchymal echo enhancement for a duration of over 7 min, whereas Definity was demonstrated to have hardly hepatic parenchymal echo enhancement [8]. In the previous report that used Definity, the post vascular phase was not observed [10]. In our study using Levovist, the contrast pattern that showed a defect in the complete tumor and an enhancement of the surrounding hepatic tissues in the post vascular phase were demonstrated in 7 of the $8 \mathrm{dogs}$, and their contrast patterns were similar to those in humans. Thus, CHI using Levovist may be clinically useful for evaluation of the post vascular phase. Moreover, in the case that a different contrast medium is used, it is suggested that a different protocol is used and the evaluation of the resultant $\mathrm{CHI}$ findings is changed in accordance with the contrast agent used. Therefore, further investigations comparing the CHI findings on using various ultrasound contrast media may be required for the standardization of $\mathrm{CHI}$ in dogs with hepatic tumors.

In our study, although the specific contrast enhancement patterns were shown in the early arterial phase and post vascular phase in canine $\mathrm{HCC}$, the $\mathrm{CHI}$ findings in the late vascular phase showed relative variations. The difference is thought to be associated with the arterial origination of the feeding vessels of HCC [1], and the various pathological changes with hypovascularity or hypervascularity in HCC [16]. In humans, it has been reported that the contrast enhancement pattern of poorly differentiated $\mathrm{HCC}$ is ambiguous in contrast to well differentiated $\operatorname{HCC}[7,15]$. Similarly, characterization of the $\mathrm{CHI}$ findings may be also difficult in the dogs with poorly differentiated HCC. In our study, the impacts of differentiation, angiogenesis and fibrosis of the tumors on different enhancement pattern were not investigated. Therefore, a clarification of the association between the pathological changes and contrast enhancement patterns may be further required for the improvement of the diagnostic quality of $\mathrm{CHI}$ in dogs with $\mathrm{HCC}$.

In conclusion, the present study showed that $\mathrm{CHI}$ was feasible in dogs and may be potentially useful for the diagnosis of canine HCC and metastatic hepatic HSA. In addition, the beginning of the $\mathrm{CHI}$ phases and characterization of the CHI findings might be similar to those of humans. Large scale studies on clinical application of CHI in dogs with hepatic tumors and hepatic diseases other than neoplasm are further required to enhance the clinical value of CHI.

\section{REFERENCES}

1. Breedis, C. and Young, G. 1954. Blood supply of neoplasms of the liver. Am. J. Pathol. 30: 969-972.

2. Dill-Macky, M. J., Burns, P. N., Khalili, K. and Wilson, S. R. 2002. Focal hepatic masses: enhancement patterns with SH U 508A and pulse-inversion US. Radiology 222: 95-102.

3. Ding, H., Kudo, M., Maekawa, K., Suetomi, Y., Minami, Y. and Onda, H. 2001. Detection of tumor parenchymal blood flow in hepatic tumors: value of second harmonic imaging with a galactose-based contrast agent. Hepatol. Res. 21: 242-251.
4. Ding, H., Kudo, M., Onda, H., Suetomi, Y., Minami, Y. and Maekawa, K. 2001. Hepatocellular carcinoma: depiction of tumor parenchymal flow with intermittent harmonic power Doppler US during the early arterial phase in dual-display mode. Radiology 220: 349-356.

5. Goldberg, B. B., Liu, J. B., Burus, P. N., Merton, D. A. and Forsberg, F. 1993. Galactose-based intravenous sonographic contrast agent; experimental studies. J. Ultrasound Med. 12: 463-470.

6. Hohmann, J., Albrecht, T., Oldenburg, A., Skrok, J. and Wolf, K. J. 2004. Liver metastases in cancer: detection with contrastenhanced ultrasonography. Abdom. Imaging 29: 669-681.

7. Isozaki, T., Numata, K., Kiba, T., Hara, K., Morimoto, M., Sakaguchi, T., Sekihara, H., Kubota, T., Shimada, H., Morizane, T. and Tanaka, K. 2003. Differential diagnosis of hepatic tumors by using contrast enhancement patterns at US. Radiology 229: 798-805.

8. Maruyama, H., Matsutani, S., Saito, H., Mine, Y., Yuki, H. and Miyata, K. 2004. Different behaviors of microbubbles in the liver: time-related quantitative analysis of two ultrasound contrast agents, Levovist and Definity. Ultrasound Med. Biol. 30: 1035-1040.

9. Nicolau, C. and Bru. C. 2004. Focal lesions: evaluation with contrast-enhanced ultrasonography. Abdom. Imaging 29: 348359.

10. O’Brien, R. T., Iani, M., Matheson, J., Delaney, F. and Young, K. 2004. Contrast harmonic ultrasound of spontaneous liver nodules in 32 dogs. Vet. Radiol. Ultrasond 45: 547-553.

11. Rabenandrasana, H. A., Furukawa, A., Furuichi, K., Yamasaki, M., Takahashi, M. and Murata, K. 2004. Comparison between tissue harmonic imaging and liver-specific late-phase contrastenhanced pulse-inversion imaging in the detection of hepatocellular carcinoma and liver metastasis. Radiat. Med. 22: 90 97.

12. Salwei, R. M., O'Brien, R. T. and Matheson, J. S. 2003. Use of contrast harmonic ultrasound for the diagnosis of congenital portosystemic shunts in three dogs. Vet. Radiol. Ultrasound 44: 301-305.

13. Shimizu, M., Iijima, H., Horibe, T., Yamada, M., Suzuki, S., Yanagisawa, K., Seki, T. and Moriyasu, F. 2004. Usefulness of contrast-enhanced ultrasonography with a new contrast mode, Agent Detection Imaging, in evaluating therapeutic response in hepatocellular carcinoma treated with radio-frequency ablation therapy. Hepatol. Res. 29: 235-242.

14. Suzuki, S., Iijima, H., Moriyasu, F., Sasaki, S., Yanagisawa, K., Miyahara, T., Oguma, K., Yoshida, M., Horibe, T., Ito, N., Kakizaki, D., Abe, K. and Tsuchiya, K. 2004. Differential diagnosis of hepatic nodules using delayed parenchymal phase imaging of Levovist contrast ultrasound: comparative study with SPIO-MRI. Hepatol. Res. 29: 122-126.

15. Suzuki, Y., Fujimoto, Y., Hosoki, Y., Suzuki, M., Sakurai, S., Ohhira, M., Saito, H and Kohgo, Y. 2003. Clinical utility of sequential imaging of hepatocellular carcinoma by contrastenhanced power Doppler ultrasonography. Eur. J. Radiol. 48: 214-219.

16. Trigo, F. J., Thompson, H., Breeze, R. G. and Nash, A.S. 1982. The pathology of liver tumors in the dog. J. Comp. Pathol. 92: 21-39.

17. Ziegler, L. E., O'Brien, R. T., Waller, K. R. and Zagzebski, J. A. 2003. Quantitative contrast harmonic ultrasound imaging of normal canine liver. Vet. Radiol. Ultrosound 44: 451-454. 